Revue internationale P.M.E.

Économie et gestion de la petite et moyenne entreprise

\title{
L'approche néo-institutionnelle et ses implications pour le management des relations avec les stakeholders en phase start-up
}

\author{
Dietmar Roessl, Matthias Fink et Sascha Kraus
}

Volume 21, numéro 3-4, 2008

État et perspectives de la recherche germanophone en entrepreneuriat et PME

URI : https://id.erudit.org/iderudit/038036ar

DOI : https://doi.org/10.7202/038036ar

Aller au sommaire du numéro

Éditeur(s)

Presses de l'Université du Québec

ISSN

0776-5436 (imprimé)

1918-9699 (numérique)

Découvrir la revue

Citer cet article

Roessl, D., Fink, M. \& Kraus, S. (2008). L’approche néo-institutionnelle et ses implications pour le management des relations avec les stakeholders en phase start-up. Revue internationale P.M.E., 21(3-4), 91-115.

https://doi.org/10.7202/038036ar
Résumé de l'article

Les approches néo-institutionnelles et surtout la théorie de l'agence et la théorie des coûts de transaction ont fait leurs preuves dans divers domaines (en marketing, en GRH, etc.). Cependant, jusqu'à présent, peu de recherches en création d'entreprise se sont appuyées sur ces approches. Le but de la présente contribution est d'analyser la création d'entreprise sous l'angle de la Nouvelle Économie institutionnelle. Cet angle souligne le rôle crucial de la relation stakeholder-créateur. En effet, le succès d'une jeune entreprise dépend directement de l'acceptation et du soutien des parties prenantes. Les relations dans la phase de création sont caractérisées par des incertitudes particulièrement fortes dues à la nouveauté (liability of newness). Dans le processus d'évaluation et de jugement de la relation d'échange, le stakeholder est confronté à l'incertitude des promesses du créateur d'entreprise ; ces incertitudes influencent la décision du stakeholder d'entrer dans la relation d'échange avec la jeune entreprise. L’aptitude à gérer ces incertitudes augmente la probabilité de succès du projet de création. L'approche MUT (Management of Uncertainties and Trust) que nous présenterons ici est un outil pragmatique et normatif qui a pour but de structurer et de résoudre la problématique d'incertitude. De cette façon, il peut favoriser le succès de nouvelles entreprises en aidant l'entrepreneur à créer des relations stables avec les parties prenantes.
Ce document est protégé par la loi sur le droit d'auteur. L'utilisation des services d’Érudit (y compris la reproduction) est assujettie à sa politique d'utilisation que vous pouvez consulter en ligne.

https://apropos.erudit.org/fr/usagers/politique-dutilisation/ 


\title{
L'approche néo-institutionnelle et ses implications pour le management des relations avec les stakeholders en phase start-up ${ }^{1}$
}

\author{
Dietmar ROESSL \\ Centre de recherche RiCC \\ Université économique de Vienne \\ Matthias FINK \\ Institut pour la gestion de la PME et Centre de recherche RiCC \\ Université économique de Vienne \\ Sascha KRAUS \\ Université du Liechtenstein et Université d'Utrecht
}

\section{Création - Incertitude - Confiance \\ Nouvelle économie institutionnelle - Stakeholder}

\begin{abstract}
LES AUTEURS
Dietmar Roessl a effectué ses études de gestion à l'Université économique de Vienne en Autriche. II a exercé dans diverses institutions dont l'Institut pour la gestion de la PME (Vienne, Autriche), l'Université de Banska Bystrica, l'Université Pilsen et l'Université libre de Bozen. En 1998, il a été nommé professeur en gestion et création d'entreprises à l'Université de Dortmund en Allemagne. Adresse: Wirtschaftsuniversität Wien, RiCC - Forschungsinstitut für Kooperationen und Genossenschaften, Augasse 2-6, A-1090 Wien, Autriche. Courriel: <dietmar.roessl@wu-wien.ac.at>.

MATTHIAS FINK a étudié la gestion à l'Université économique de Vienne en Autriche. Aujourd'hui, il y est professeur assistant et membre de l'Institut pour la gestion de la PME. II est également chercheur sénior au RiCC. II a obtenu une bourse APART (Austrian Programme for Advanced Research and Technology) de l'Académie des sciences autrichienne pour financer son habilitation à diriger les recherches. II est membre fondateur de l'Institut de recherche en Management (Cologne) et vice-président pour l'Autriche au European Council for Small Business (ECSB). Adresse: Wirtschaftsuniversität Wien, RiCC - Forschungsinstitut für Kooperationen und Genossenschaften, Inst. F. Betriebswirtschaftslehre der Klein- und Mittelbetriebe, Augasse 2-6, A-1090 Wien, Autriche. Courriel: <matthias.fink@wu-wien.ac.at>.
\end{abstract}

1. Les auteurs remercient chaleureusement Ilse Roessl pour la traduction méticuleuse. 
SASCHA KRAUS est professeur en entrepreneuriat à l'École supérieure du Liechtenstein, ainsi qu'à I'Université d'Utrecht aux Pays-Bas. II a exercé dans diverses universités dont l'Université de Vaasa, en Finlande, l'École supérieure de Salzbourg en Autriche, l'Université de Klagenfurt en Autriche et l'Université d'Oldenburg en Allemagne. Il a effectué ses études de gestion à l'Université de Cologne, à l'Université de Siegen, en Allemagne, comme à l'Université de Southern Queensland en Australie. Depuis 2008, il est vice-président pour le pays au European Council for Small Business (ECSB). Adresse: University of Liechtenstein, Fürst-Franz-Josef-Strasse, LI-9490 Vaduz, Liechtenstein. Courriel: <sascha.kraus@ hochschule.li>.

\title{
RÉSUMÉ
}

Les approches néo-institutionnelles et surtout la théorie de l'agence et la théorie des coûts de transaction ont fait leurs preuves dans divers domaines (en marketing, en $\mathrm{GRH}$, etc.). Cependant, jusqu'à présent, peu de recherches en création d'entreprise se sont appuyées sur ces approches. Le but de la présente contribution est d'analyser la création d'entreprise sous l'angle de la Nouvelle Économie institutionnelle. Cet angle souligne le rôle crucial de la relation stakeholder-créateur. En effet, le succès d'une jeune entreprise dépend directement de l'acceptation et du soutien des parties prenantes. Les relations dans la phase de création sont caractérisées par des incertitudes particulièrement fortes dues à la nouveauté (liability of newness). Dans le processus d'évaluation et de jugement de la relation d'échange, le stakeholder est confronté à l'incertitude des promesses du créateur d'entreprise; ces incertitudes influencent la décision du stakeholder d'entrer dans la relation d'échange avec la jeune entreprise. L'aptitude à gérer ces incertitudes augmente la probabilité de succès du projet de création. L'approche MUT (Management of Uncertainties and Trust) que nous présenterons ici est un outil pragmatique et normatif qui a pour but de structurer et de résoudre la problématique d'incertitude. De cette façon, il peut favoriser le succès de nouvelles entreprises en aidant l'entrepreneur à créer des relations stables avec les parties prenantes.

\begin{abstract}
Institutional Economics, especially the Principal-Agent Approach and Transaction Cost Economics, has proven of value in various disciplines (e.g. marketing, personnel management). However, so far they have hardly expanded into entrepreneurship research. In this paper, we strive to analyse the establishment of new ventures from the perspective of Institutional Economics. This highlights the decisive role of the relationship between the founder and the stakeholders. The success of a new venture strongly depends on the acceptance and the support of the stakeholders. At the same time, the relationships to the stakeholders are burdened with particularly high uncertainties, because of the "liability of newness." When evaluating the offered transaction, the stakeholder is confronted by the founder's insecure promises. These insecurities influence the potential stakeholders' decision whether or not to establish a transaction relationship with the newly founded company. With the rising capability to handle such insecurities, the success of the new venture becomes more likely. As a pragmatic-normative tool to structure and handle the insecurities identified, the MUT-Approach presented in this paper can foster the success of new ventures by helping the founder to establish stable relationships with stakeholders.
\end{abstract}




\section{RESUMEN}

Los enfoques de la Economía Institucional, en particular la teoría del AgentePrincipal y la de los costos de la transacción, han demostrado ser muy útiles en diversas disciplinas (por ejemplo marketing, gestión de personal). Sin embargo, hasta ahora no se han ampliado tanto en la investigación de la iniciativa empresarial. En este artículo nos esforzamos en analizar la creación de nuevas empresas desde la perspectiva de la Economía Institucional. Esto pone de relieve el papel decisivo de la relación entre el fundador y los interesados. El éxito de una nueva empresa depende en gran medida de la aceptación y el apoyo de las partes interesadas. Al mismo tiempo, estas relaciones son particularmente cargadas de grandes incertidumbres debido a la novedad (liability of newness). Al evaluar la transacción ofrecida, el interesado es confrontado con las promesas inseguras del fundador. Estas inseguridades influyen en la decisión de las potenciales partes interesadas de establecer o no una relación de intercambio con la recién creada empresa. El éxito de la nueva empresa se hace más probable con el aumento de la capacidad de manejar esas inseguridades. El MUT-Enfoque (Management of Uncertainties and Trust) presentado en este documento es un instrumento pragmático y normativo para estructurar y manejar el problema de las inseguridades identificadas. De esta forma puede favorecer el éxito de nuevas empresas, ayudando al fundador a establecer relaciones estables con las partes interesadas.

\section{ZUSAMMENFASSUNG}

Die Ansätze der Institutional Economics insbesondere Prinzipal-Agent-Theorie und Transaktionskostentheorie haben sich in unterschiedlichsten Fachbereichen (z.B. Marketing, Personalmanagement, etc.) bewährt. Haben. In der Entrepreneurship-Forschung haben diese Ansätze aber bislang wenig Eingang gefunden. Dieser Beitrag setzt sich zum Ziel, die Unternehmensgründung aus der Perspektive der Institutional Economics zu analysieren. Dies eröffnet den Blick auf die erfolgskritische Rolle der Stakeholder-Gründer-Beziehung. Die Beziehungen in der Gründungsphase sind aufgrund der liability of newness mit besonders hohen Unsicherheiten behaftet. Mit der Kompetenz, die Unsicherheiten zu handhaben, steigt die Erfolgswahrscheinlichkeit der Gründung. Der hier präsentierte MUT-Ansatz (Management of Uncertaintites and Trust) ist ein pragmatisch-normativer Ansatz zur Strukturierung und Lösung der Unsicherheitsproblematik. 


\section{Introduction}

La gestion des relations entre le créateur d'entreprise et les parties prenantes $(\text { stakeholder })^{2}$ comme facteur clé du succès n'a été examinée qu'implicitement dans diverses approches théoriques (par exemple, dans la «resource dependence theory» ou la «population ecological approach»). Chez Low et Srivatsan (1995) et Gruber (2004), le défi de la gestion des relations avec les stakeholders est traité explicitement. Kollmann et Kuckertz (2006b) examinent cette problématique dans le domaine des relations avec les investisseurs. Dans le contexte de l'évaluation des créations d'entreprises, le modèle Powell et Bimmerle (1980) se réfère aussi aux éléments de soutien financier, psychologique et technique des relations avec les stakeholders.

Barnard (1938), puis March et Simon (1958) ont mis au jour la perspective des stakeholders dans le modèle de coalition d'entreprise et Freeman (1984) l'a perfectionnée. Dans l'approche «corporate social responsibility» (CSR), l'analyse de la problématique des stakeholders se fait sous l'angle de la tension entre responsabilité sociale et succès économique et vise le management des entreprises. Comprise ainsi, cette approche risque de perdre son pouvoir analytique. Contrairement à Frederick, Post et Davis (1992) et à Preston, Donaldson et Brooks (1999), nous ne comprenons pas l'approche des parties prenantes comme une approche basée sur des considérations normatives éthiques, mais plutôt comme une approche ayant pour but de renforcer la puissance de l'entreprise sur le plan concurrentiel.

Dans la perspective de la «instrumental stakeholder theory» (Jones, 1995), la gestion active des relations avec les parties prenantes est importante puisqu'elle garantit la survie de l'entreprise à long terme (voir Freeman et McVea, 2005) ${ }^{3}$. Cette vision correspond aux arguments des tenants de l'approche stakeholder qui soutiennent que la prise en compte des stakeholder est une condition nécessaire pour maximiser la «shareholder value». De plus, elle correspond à l'observation voulant que le succès économique et la réa-

2. Dans ce contexte, les groupes, individus ou organisations, qui peuvent entraver ou aider à atteindre l'objectif d'une organisation sont nommés stakeholders (Phillips, Freeman et Wicks, 2003, p. 481). Puisqu'ils peuvent mettre à disposition des ressources ou bien les retirer, ils sont capables d'influencer le comportement de l'entreprise. Les stakeholders peuvent gagner ou perdre en fonction du comportement de l'entreprise (Preston, Donaldson et Brooks, 1999, p. 2). À notre avis, la limitation de la notion de stakeholder aux groupes sans le soutien desquels l'organisation cesserait d'exister (Freeman, 1984, p. 31) est trop restreinte.

3. Au sujet des interprétations et «mauvaises » interprétations de l'approche stakeholder, voir Freeman et Phillips (2002) et Phillips, Freeman et Wicks (2003). Pour le rapport entre CSR et approche stakeholder, voir Wheeler, Colbert et Freeman (2003). 
lisation des intérêts des parties prenantes soient en corrélation (Rappaport, 1995), comme le confirment les résultats de Berman et al. (1999): «support the idea that managerial attention to multiple stakeholder interests can affect firm financial performance, providing concrete support for an argument long advanced by stakeholder theorists ». Alors que l'analyse des relations avec les parties prenantes donne un cadre global pour le développement et la mise en œuvre d'une stratégie, l'approche shareholder-value propose des critères appropriés pour l'évaluation et le monitoring.

Ces dernières décennies, des progrès considérables ont été réalisés dans les domaines de la théorie des coûts de transaction et de la théorie de l'agence (Williamson, 2000). Ces progrès ont stimulé l'analyse des questions managériales concernant les relations d'échange avec les stakeholders. Comme ces approches théoriques mettent autant l'accent sur l'analyse des incertitudes et la possibilité de les maîtriser que sur les relations d'échange elles-mêmes, et que ces aspects sont très importants dans la phase de start-up, l'application de cette perspective au management dans cette phase mérite d'être prise en considération.

Étonnamment, Fallgatter (2004) réduit le problème principal-agent aux problèmes managériaux des propriétaires et doute, par conséquent, de l'applicabilité de cette approche à la problématique de la création d'entreprise. Pour le succès des nouvelles entreprises, les avantages résultant des coûts de transaction sont importants, c'est pourquoi une prise en compte précoce de ces coûts s'impose. Certes, le résultat d'une telle analyse ne détermine pas l'intention de créer l'entreprise. Cependant, une telle perspective, au sens d'une approche heuristique, peut décider de la manière dont l'idée de création va être réalisée. Nous convenons avec Fallgatter (2004) que ces réflexions vues sous l'angle de la théorie des coûts de transaction ne peuvent pas constituer un facteur décisif de création mais, à notre avis, cette théorie peut être appliquée pour analyser comment quelqu'un peut créer une entreprise et comment il peut la faire croître. Cette position est également illustrée par l'étude de Brouthers et Nakos (2004).

Les entreprises agissent dans un réseau de relations avec des stakeholders importants. Les créateurs d'entreprises dépendent du soutien et de l'acceptation des fournisseurs, des investisseurs, des clients, des employés, des voisins, etc., et ils se trouvent en concurrence pour le soutien de ces groupes avec d'autres entreprises déjà existantes. «The lack of exchange relationships of new ventures is challenging not only in the context of customers, but also for other parties such as distributors and suppliers » (Gruber, 2004). Les résultats empiriques de Kollmann et Kuckertz $(2005,2006 a)$ montrent que la date de création de l'entreprise dépend souvent du comportement des stakeholders 
potentiels. La nécessité d'obtenir le soutien des stakeholders, dont les intérêts sont souvent divergents, constitue autant un problème crucial qu'une chance formidable pour les jeunes entreprises:

- C'est une chance puisque, finalement, le succès de l'entreprise dépend de la participation des stakeholders au projet du créateur. Vu sous cet angle, le succès du créateur est une prophétie autoréalisatrice (Low et Srivatsan, 1995): convaincre les acteurs intéressés qu'on est en train de créer une entreprise compétitive à long terme et que la relation d'échange offerte est par conséquent attirante (ou du moins peut l'être) est fondamental pour le succès de la jeune entreprise. C'est seulement si les partenaires potentiels laissent leurs doutes de côté et acceptent de prendre le risque lié à l'incertitude de la relation d'échange que la «venture» réussira.

- C'est un problème, puisque l'entrepreneur devrait être «tout pour tous »: employeur loyal pour les employés, citoyen respectant les lois, responsable envers l'environnement pour les voisins et la société, entrepreneur soucieux de satisfaire au mieux les besoins de ses clients, entrepreneur maximisant le profit pour les investisseurs, etc.

Chaque entreprise doit pouvoir offrir des relations d'échange avantageuses à ses partenaires. Cela pose un véritable problème dans la phase de start-up: le savoir minimal que le principal (le stakeholder) possède de l'agent (le créateur) renforce la problématique d'asymétrie d'information. Ainsi, l'importance de la relation de confiance entre les partenaires augmente (Kollmann et Kuckertz 2006b). En raison de la nouveauté de l'entreprise, il est impossible de prévoir le comportement du créateur, en conséquence, les parties prenantes ne peuvent pas développer des attentes. Le créateur dispose alors d'une large marge de manœuvre discrétionnaire, accroissant ainsi le danger d'un comportement opportuniste (Grichnik et Schwaerzel, 2005). Aux problèmes généraux principal-agent (voir, par exemple, Kleinaltenkamp et Jacob, 2002) s'ajoute la question de savoir si le projet de création sera un succès (Gruber, 2004) et si l'engagement sera profitable pour le stakeholder (Kollmann et Kuckertz, 2006b). Cela est encore plus vrai dans le cas des jeunes entreprises fortement innovantes (Grichnik et Schwaerzel, 2005). Le succès d'une entreprise dépend ainsi énormément de l'aptitude de l'entrepreneur à gagner le soutien des autres partenaires (investisseurs, consommateurs, fournisseurs, etc.; Low et Srivatsan, 1995). Le stakeholder potentiel doit décider de sa participation au projet de création sans connaître 
la décision des autres stakeholders, qui sont aussi déterminantes pour le succès de l'entreprise (Gaechter, Herrmann et Thoeni, 2004). Par exemple, un employé potentiel doit décider de l'offre d'emploi sans connaître le futur succès de la jeune entreprise. Ainsi, le succès de la start-up dépend essentiellement de la volonté des partenaires potentiels à entrer dans la relation d'échange incertaine offerte par l'entrepreneur. Le fardeau de nouveauté (liability of newness) perçu par les stakeholders semble être négativement lié à la décision de s'engager dans une nouvelle entreprise (Choi, 2001). Par conséquent, la question suivante se pose: Comment un entrepreneur qui ne peut pas recourir à une tradition, à des références, etc., peut-il obtenir le soutien des stakeholders?

Pour traiter cette problématique, nous procéderons dans la présente étude en plusieurs temps. Dans un premier temps, la relation stakeholdercréateur est analysée et les incertitudes du stakeholder sont relevées, ce qui soulèvera la question suivante: Comment le créateur peut-il réduire ces incertitudes? Dans un deuxième temps, la théorie des coûts de transactions est appliquée, afin de montrer que ces incertitudes ne peuvent pas être dissipées uniquement par des instruments hiérarchiques; cela souligne l'importance de la confiance comme mécanisme coordinateur. Dans un troisième temps, pour montrer comment le créateur d'entreprise peut convaincre ses stakeholders potentiels de ses qualifications et de sa fiabilité, nous appliquerons l'approche MUT (Management of uncertainties and trust). Finalement, en conclusion, nous dégagerons des mesures concrètes pour maîtriser les incertitudes dans la phase du start-up.

\section{Les caractéristiques des biens échangés}

L'analyse des objets d'échange fournis par les jeunes entrepreneurs, examinés selon les aspects du concret et de l'unique (concreteness/uniqueness) (figure 1), montre la forte incertitude du côté des stakeholders. Dans ce contexte, nous examinons les relations potentielles entre les créateurs et les stakeholders. La notion du «bien échangé» ne se limite pas aux produits matériaux ou à une prestation de service, mais comprend aussi des postes de travail offerts, des possibilités d'investissement, etc. 
FIGURE 1

Caractéristiques des biens échangés et les incertitudes résultantes

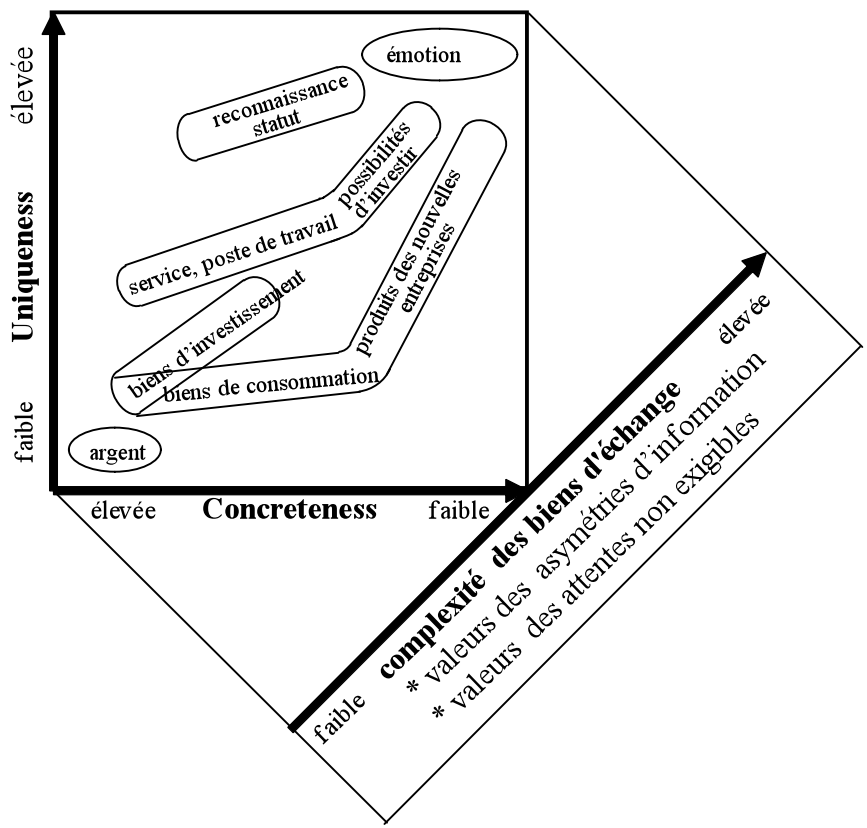

Source: Roessl, 2006.

La notion de «concreteness» fait référence à la capacité ex ante de définir le bien échangé, à la possibilité de sa détermination contractuelle ou à son contrôle préliminaire avant la conclusion de la transaction. Une «concreteness » faible signifie que le bien échangé doit être caractérisé plutôt comme «promesse ( Kaas, 1992a; Levitt, 1981).

La notion d'«uniqueness » renvoie à l'influence des circonstances spécifiques et de l'identité des partenaires de transaction sur l'évaluation du bien échangé par le destinataire de la performance (Griesinger, 1990; Dwyer, Schurr et Oh,1987). Ainsi, la notion d'«uniqueness» reflète la spécificité des biens échangés (par exemple, Picot, 1991) et implique une «small-numberexchange-situation» (Picot, 1991). «Asset specificity means that a tangible or intangible asset has value in a particular environment but loses at least some of this value in other contexts » (Kleinaltenkamp et Jacob,2002), ce qui implique des dépendances (Williamson, 2005; 2003). Les transactions avec des startups innovantes se distinguent par une telle grande spécificité en raison de 
la nouveauté ( «uniqueness » élevée) et du large éventail de comportements possibles (faible «concreteness»; Grichnik et Schwaerzel, 2005; Brouthers et Nakos, 2004).

En considérant ces aspects, voici nos constats :

- Les relations d'échange entre les créateurs et les stakeholders potentiels concernent souvent des biens qui n'existent pas encore au moment de l'accord (Fallgatter, 2004) et qui possèdent donc moins de «search qualities» que d' «experience et de credence qualities» (Weiber et Adler, 1995). En conséquence, il ne suffit pas de préciser les éléments de performance: on doit développer la crédibilité des attentes (Kaas, 1992b). De fortes incertitudes exogènes en résultent (Grichnik et Schwaerzel, 2005).

- Les relations d'échange entre les créateurs et les stakeholders potentiels visent souvent des performances qui ne peuvent ou ne doivent pas encore être fixées entièrement au moment de la conclusion du contrat; ainsi, il est nécessaire de conclure des accords cadres, qui permettraient une adaptation flexible aux nouveaux développements (Hauschildt et Leker, 1990).

- Les relations d'échange entre les créateurs et les stakeholders potentiels se réfèrent souvent à des performances individuelles, que l'on peut difficilement évaluer, faute de possibilités de comparaison.

- Les relations d'échange entre les créateurs et les stakeholders potentiels ont souvent trait à des performances ayant une faible fréquence de transaction et/ou un prix élevé et/ou une longue durée de vie du produit, rendant ainsi l'effet d'expérience ou l'apprentissage par erreurs difficile pour les deux parties (par exemple, la construction d'une maison). Toutefois, après un certain laps de temps, il existe assez d'expériences sur lesquelles on peut s'appuyer (Gierl et Bambauer, 2002).

Le stakeholder potentiel ne peut examiner que quelques-unes des caractéristiques de l'objet d'échange offert par le créateur d'entreprise. Ce manque de possibilités d'évaluation a priori résulte:

- le décalage entre le temps de l'accord, et celui de la production et/ ou de la réalisation de l'avantage de l'échange (par exemple, si un outil satisfait ou non les attentes ne se manifeste que lors de son utilisation), et/ou 
- du manque de savoir-faire du stakeholder et du manque de possibilités de contrôle des performances réalisées par le jeune entrepreneur (par exemple, le client ne peut pas juger la performance d'un plombier quand il n'est pas expert lui-même).

Dans la mesure où les caractéristiques de la relation d'échange ne peuvent pas être évaluées avant l'entrée en relation, les incertitudes augmentent pour le stakeholder potentiel. L'acceptation de ces incertitudes doit être envisagée comme une contribution supplémentaire du stakeholder (en plus, par exemple, du prix du bien échangé). Donc, d'une part, plus les incertitudes sont élevées, moins la transaction semble intéressante pour le stakeholder (par exemple, si, en plus du prix, un client est confronté à de fortes incertitudes, la transaction semble peu intéressante). D'autre part, de fortes incertitudes de la part du stakeholder permettent au créateur de prendre des mesures pour réduire les incertitudes et d'offrir de la «crédibilité», ce qui peut lui procurer des avantages concurrentiels par rapport à d'autres entreprises.

\section{La théorie des coûts de transaction et la maîtrise de l'incertitude}

Dans ce contexte d'incertitude, une question se pose: Comment créateur et stakeholder potentiel peuvent-ils surmonter ces incertitudes paralysantes afin que cette relation puisse naître? Cette relation peut avoir une importance existentielle pour le créateur et peut fournir des avantages économiques au stakeholder. Si cette transaction suit les mécanismes du marché, les incertitudes du stakeholder, concernant le comportement du jeune entrepreneur, sont remplacées par la certitude que ce dernier recherchera - conformément au calcul de son avantage à court terme - son avantage et qu'il exploitera chaque possibilité d'opportunisme. Cependant, dans cette optique, la relation offerte est rendue impossible par le fait qu'elle demande certaines préperformances de la part du stakeholder (par exemple, accorder un crédit) qui ne sont pas justifiées à cause de l'opportunisme «illimité» du créateur. Jones (2005) qualifie cette incertitude de «bounded rationality» comme une chance pour le créateur d'entreprise, car c'est seulement ainsi que des innovations et des actions entrepreneuriales au sens de Schumpeter se révèlent indispensables.

D'après la théorie des coûts de transaction (figure 2), la gouvernance hiérarchique, particulièrement dans le cas de transactions complexes avec des investissements spécifiques, se révèle être nécessaire. Dans un cas extrême, il 
en résulte l'intégration de l'entreprise (Williamson, 2003). Cependant, deux arguments peuvent être opposés à cette intégration. Premièrement, l'intégration des ressources ne fait que déplacer le problème, puisque la fabrication interne des ressources exige des relations d'échange avec d'autres stakeholders, donc de nouveaux investisseurs, employés, etc., doivent être gagnés au projet. Cela implique des coûts de contrôle internes plus élevés (Brouthers et Nakos, 2004). Deuxièmement, comme nous l'avons relevé plus haut, les relations créateur-stakeholder ne sont ni complètement programmables ni contrôlables ex post. Par conséquent, tenter de maîtriser les incertitudes par des accords intégraux et par un contrôle hiérarchique total - contingent claim contracts -, entraîne, d'une part, des coûts de transactions élevés et, d'autre part - c'est le cœur du problème -, ne peut pas faire disparaître les incertitudes (Roessl, 1996).

\section{FigURE 2}

\section{Les fonctions des coûts de transaction}

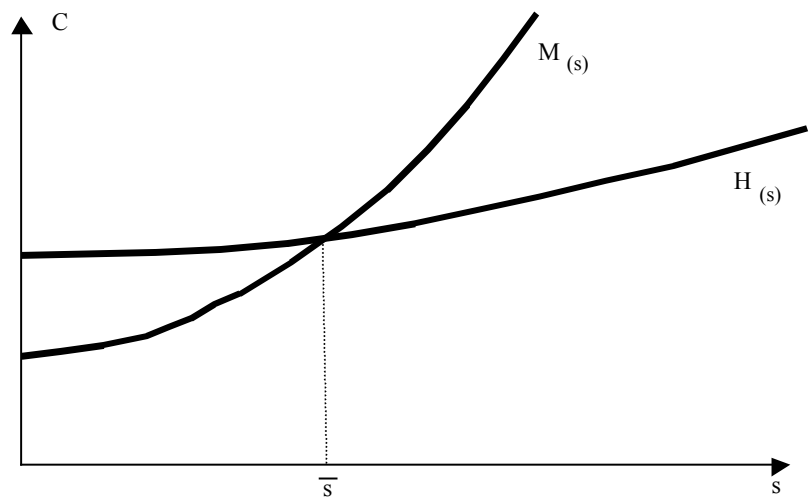

C coûts de transaction

M mécanismes de marché

$\mathrm{H}$ hiérarchie

s complexité/spécificité

Sources: Williamson $(1991,2003)$.

- Prenant les incertitudes de planification en compte, le comportement souhaité n'est pas toujours déterminable ex ante. Un contrat complet ne peut pas être formulé à partir d'une information incomplète 
(Williamson, 2002; Becker, 2001; Schrader,1993;Kaas, 1992b;Provan et Skinner, 1989). Des contrats incomplets et le manque de contrôle du comportement nécessitent la coopération volontaire des agents économiques (Gaechter, Herrmann et Thoeni, 2004).

- Si l'on ne peut pas prévoir avec certitude le résultat de la performance (par exemple, dans le cas d'influences externes inconnues), on ne peut pas établir de sanctions basées sur le résultat, ce qui réduit considérablement leur fonction de gouvernance du comportement (Kaas, 1992b). Des sanctions crédibles présupposent la connaissance ex ante du comportement souhaité (Eberl, 2004) et l'observation ex post du comportement réalisé (Gierl, 2001).

- Dans les cas où l'importance de la transaction exige un certain résultat, des sanctions ex post n'ont aucun sens. Par exemple, un «business angel» peut priver le créateur de son soutien, mais après l'échec de l'entreprise et la perte du capital, cette sanction ex post est peu pertinente.

Ainsi, nous pouvons constater que la gouvernance hiérarchique ne peut pas résoudre la problématique des relations avec les stakeholders à cause de l'échec des mécanismes organisationnels (Semlinger, 1993).

\section{Surmonter les incertitudes dans l'approche MUT}

\subsection{L'approche MUT}

En général, la théorie des coûts de transaction suppose que les incertitudes ne peuvent être éliminées - quoiqu'elles puissent être accompagnées de coûts de transaction - que par la gouvernance hiérarchique, et non pas par une relation de confiance. Eberl (2004), renvoyant à l'évidence empirique des relations basées sur la confiance, s'oppose à la position de Williamson selon laquelle de telles relations seraient une utopie (Williamson, 1981; Fink, 2005 ; Roessl, 1996). Adler (2001) rejette la dichotomie qui oppose marché et hiérarchie et partage l'avis de Roessl (1996), qu'il existe trois mécanismes de coordination (marché, hiérarchie et confiance), qui apparaissent sous diverses combinaisons (Carson, Madhok et Wu, 2006; Fink, 2005; Eberl, 2004; Frey et Osterloh, 2002; Sydow et Windeler, 2000; Roessl, 1996). Du point de vue de l'économie d'information, la réduction des incertitudes ou la création de confiance sont des équivalents fonctionnels, puisque au sein d'une relation de confiance, une marge de comportement peut être acceptée (Spremann, 1990). 
Dans l'approche $\mathrm{MUT}^{4}$ (figure 3), la tentative de faire disparaître les incertitudes du stakeholder par la réduction de possibilités de comportements opportunistes (utilisation d'instruments de coordination hiérarchiques) est complétée par une réduction de la tentation à l'opportunisme (création de confiance). Ainsi, les risques de la relation d'échange offerte sont d'autant plus réduits que les stakeholders potentiels sont encouragés à entrer dans la relation d'échange toujours incertaine. Cela peut être réalisé, par exemple, sur la base de la réputation du créateur comme businessman digne de confiance. La perception du risque se réduit aussi avec l'expérience que, jusqu'à présent, le partenaire de transaction n'a pas exploité la marge d'opportunisme (Siegrist, 2001).

La création d'un lien de confiance ne devient importante que lorsque les mécanismes de coordination hiérarchiques ne sont plus possibles ou efficients. "Conditions for using trust-based self-coordination are only given if task ambiguity is high, and measurability of job performance is low» (Eberl, 2004; Harvey, 2002). Si les incertitudes ne sont pas trop importantes ou si elles sont surmontables par des instruments de coordination hiérarchiques ou par la création d'un lien de confiance, la relation est déjouée ou elle peut être - si, malgré les fortes incertitudes, les participants perçoivent des avantages - cédée aux mécanismes de marché.

Ces considérations ne sont pas destinées à suggérer ici un processus de prise de décision conscient et cognitif de la part du créateur. Le modèle est plutôt une structuration idéale au sens d'un modèle théorique. Le modèle montre également comment un créateur devrait aborder le problème du management des relations avec les stakeholders. Le modèle résulte logiquement de l'interdépendance des coûts du risque (qui résultent de la marge d'opportunisme ou de la tendance à l'opportunisme) et des coûts des instruments pour la réduction de la marge d'opportunisme ou de la tendance à l'opportunisme. Cependant, d'autres analyses - notamment des analyses empiriques - sont nécessaires.

4. L'approche MUT ou Management of uncertainties and trust a été conçue comme une solution pragmatique normative afin de gérer des relations de coopération dyadiques. L'approche combine le marketing comme instrument pour l'analyse des relations d'échange avec les bases théoriques de l'évolution de coopération (théorie des coûts de transaction, théorie de l'agence, théorie des systèmes; Roessl, 1994). 


\section{Figure 3}

\section{La réduction de l'incertitude versus la confiance dans l'approche MUT}

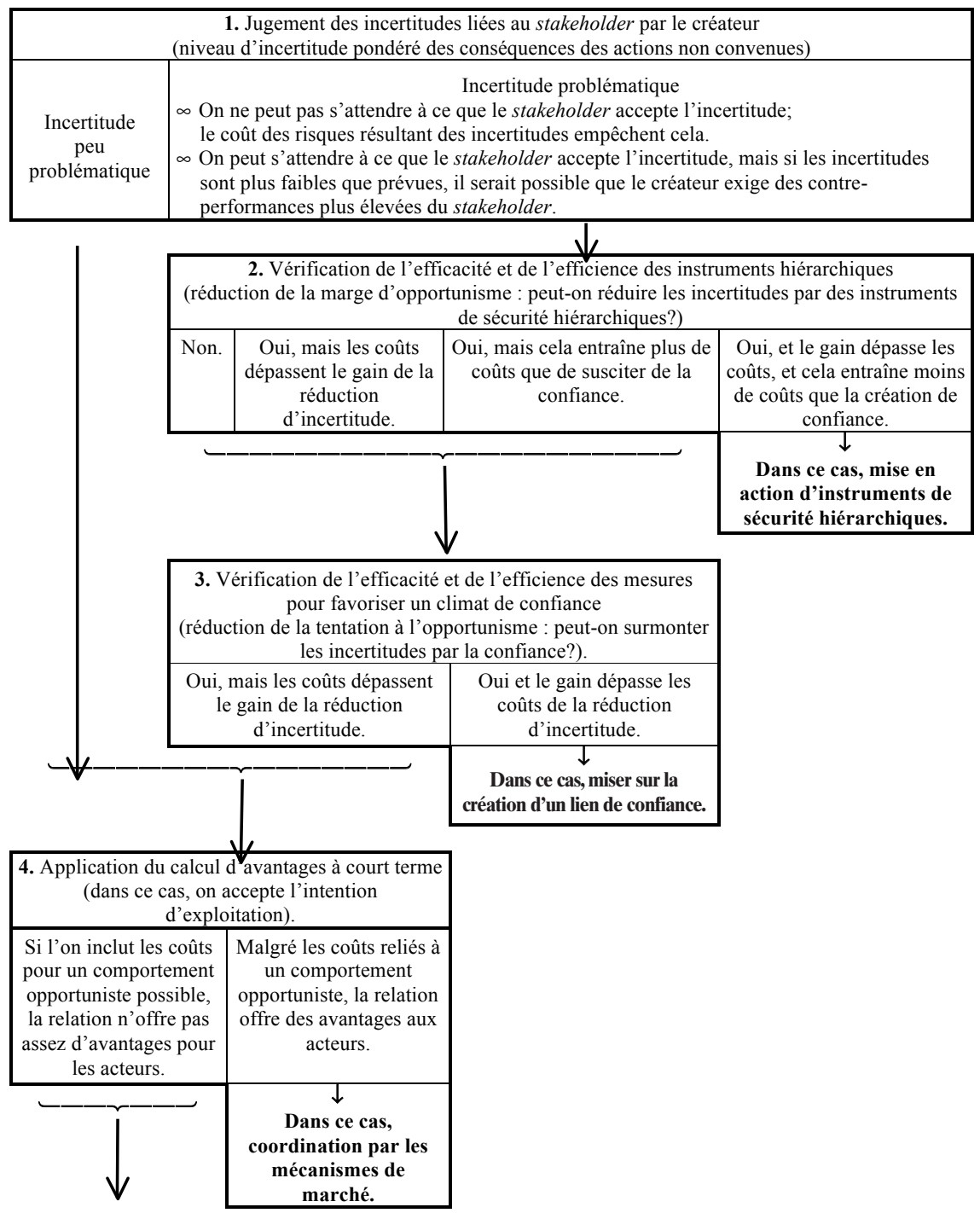

\footnotetext{
Une relation créateur-

stakeholder ne naît

pas.
} 


\subsection{Le management des relations avec les stakeholders selon l'approche MUT}

S'il y a accord avec le créateur, les stakeholders s'exposent au risque que le créateur ne soit pas aussi performant qu'ils s'y attendaient. Pour que la relation d'échange soit intéressante pour le stakeholder, il faut qu'il puisse être sûr que le jeune entrepreneur n'exploite pas les possibilités d'opportunisme. Cela peut se faire selon deux manières:

- Par des mécanismes de contrôle hiérarchiques. Ainsi, les possibilités d'opportunisme du jeune créateur sont limitées.

- Par la création de confiance mutuelle. Ainsi, le stakeholder a une certitude suffisante que le jeune entrepreneur renoncera à son calcul d'avantage à court terme et n'exploitera pas les possibilités d'opportunisme à court terme.

Comme le jeune entrepreneur a besoin de soutien de la part du stakeholder, c'est à lui d'éliminer les sources d'incertitudes pour ce dernier, de les relativiser et/ou de communiquer de manière crédible qu'il est digne de confiance. Le créateur doit prendre des mesures pour que son projet soit attirant pour les stakeholders, dont le soutien est essentiel au succès du projet de création. Ainsi, les facteurs qui entrent en jeu sont, premièrement, une réduction des incertitudes pour les stakeholders potentiels - tout en s'assurant que les coûts de cette réduction restent peu élevés - et, deuxièmement, la création de confiance; particulièrement importante dans le cas des incertitudes qu'on ne peut réduire qu'à des coûts exorbitants.

\subsubsection{La réduction des incertitudes des stakeholders}

Un créateur d'entreprise, qui n'a pas encore de réputation et qui ne peut pas s'appuyer sur son histoire pour prouver qu'il est digne de confiance (développement de l'entreprise, succès sur le marché, etc.), doit prendre des mesures pour réduire les incertitudes des stakeholders autrement perçues comme trop fortes (Kollmann et Kuckertz, 2006b). Cependant, les arguments suivants s'opposent à la réduction des incertitudes :

- Les coûts élevés de la réduction des incertitudes (Breid,1995; Kaas, 1992b) laissent apparaître la création de confiance comme une alternative intéressante. "So relatively, trust is the more efficient option because it economizes on time and information processing requirements » (Jones, 2005). «Trust can dramatically reduce both transaction costs - replacing contracts with handshakes - and agency risks replacing the fear of shirking and misrepresentation with mutual 
confidence» (Adler, 2001). Par exemple, s'il y a sous-performance du créateur et qu'un dommage énorme en résulte pour le stakeholder, des garanties de performance ne peuvent limiter efficacement les incertitudes que si elles impliquent des paiements de compensation très élevés (Spremann, 1988).

- De plus, on ne peut pas réduire efficacement toutes les incertitudes. Par exemple, des garanties sur le résultat ne peuvent pas réduire les incertitudes lorsque les performances du créateur ne sont pas contrôlables, lorsque la sous-performance se manifeste avec un grand retard ou lorsqu'on ne peut pas mettre en relation en toute certitude le résultat avec les causes. Ainsi, si l'on ne peut pas établir si la sous-performance du créateur est due aux influences défavorables externes, à sa mauvaise qualification ou à un manque d'effort de la part du créateur, des sanctions sont inefficaces. De plus, aucune mesure ne peut faire disparaître entièrement les incertitudes du stakeholder quant à la volonté de performance du créateur. Il est impossible d'empêcher le créateur de vouloir réaliser une sous-performance. Cela est particulièrement valable lorsqu'une telle sous-performance reste cachée aux yeux du stakeholder (Kleinaltenkamp et Jacob, 2002; Kleinaltenkamp, 1992; Kaas, 1992a).

- Finalement, la réduction de l'incertitude n'est pas toujours dans l'intérêt du créateur. Une réduction de l'incertitude renforce la possibilité de régler la transaction contractuellement à cause de la «concreteness» élevée. Parallèlement, la confiance perd de son importance dans la transaction et le créateur peut être plus facilement remplacé par des compétiteurs (Kleinaltenkamp, 1992; Kaas, 1992a). Les créateurs, en tant qu'agents sans antécédents auxquels on puisse se référer, ont intérêt à augmenter les informations disponibles aux stakeholders - à condition qu'ils n'aient pas l'intention d'exploiter les incertitudes et qu'ils produisent en effet la performance convenue. Si la relation d'échange est établie et des premiers signes de confiance existent, les créateurs d'entreprise essaient de rétablir des asymétries d'information (par exemple, par des innovations), afin que le capital-confiance dans la relation garde la même valeur (Kleinaltenkamp, 1992).

\subsubsection{La création de confiance dans la relation stakeholder-créateur}

En plus des mesures prises en vue d'une réduction des incertitudes, il est possible de prendre des mesures pour la création de confiance afin de convaincre les stakeholders potentiels de l'intérêt de la relation 
d'échange offerte. Le management de confiance vise à influencer les éléments qui permettent au stakeholder d'évaluer si le créateur est digne de confiance:

- le savoir du stakeholder quant au passé et à l'évolution du créateur d'entreprise;

- la vraisemblance de cette information et des sources d'information correspondantes;

- la démonstration des principes comportementaux stables de la part du créateur, qui permet à la confiance de s'établir. En effet, le stakeholder ne peut accorder sa confiance que si le comportement du créateur correspond à long terme à des principes comportementaux stables (Luhmann, 1989; Lindskold, 1981).

Dans le cadre d'une stratégie de réputation, l'entrepreneur essaie de convaincre les stakeholders qu'il est un partenaire digne de confiance, en leur communiquant de façon crédible qu'il se comporte de manière convenue et, de ce fait, conformément à sa réputation. Avec un comportement engendrant la confiance, il continue d'écrire son histoire de manière consistante et crée ainsi des éléments nouveaux témoignant qu'il est une personne digne de confiance (Gierl et Gehrke, 2004).

\section{Conclusions}

Sur la base de nos considérations en termes de théorie de l'agence et de théorie des coûts de transactions, nous pouvons maintenant présenter les actions que nous recommandons de réaliser au plan pragmatique normatif.

\section{Les mesures pour la réduction des incertitudes des stakeholders}

Premièrement, le créateur peut démontrer sa compétence à juger les stakeholders. La sélection des partenaires compétents et appropriés permet au créateur d'entreprise de démontrer sa qualification. Cette qualification et la manière soigneuse avec laquelle l'entrepreneur sélectionne ses partenaires sont des indicateurs du succès probable de l'entreprise créée. Les stakeholders potentiels se poseront la question de savoir si le créateur évalue systématiquement la fiabilité du stakeholder ou s'il lui accorde sa confiance sans faire de vérifications.

Deuxièmement, le créateur peut démontrer sa compétence à gérer les relations avec les stakeholders. S'assurer des performances promises par les stakeholders n'est pas uniquement dans l'intérêt du créateur. La capacité et 
la volonté montrée du créateur de contre-attaquer effectivement en cas de comportement défectueux d'un stakeholder réduisent le risque du créateur d'être exploité. Pour les partenaires d'échange potentiels, la diligence avec laquelle le créateur assure la réalisation des performances qui lui ont été promises est un autre indicateur de probabilité du succès de la jeune entreprise. Les stakeholders sont conscients que le créateur dépend simultanément de beaucoup de partenaires. Si le créateur fait des efforts démesurés pour gagner le soutien d'un stakeholder en particulier, celui-ci peut se demander si le créateur cherche à faire croire à chaque stakeholder qu'il va se vouer complètement aux intérêts de chacun d'entre eux (chameleonic figure) ou si le créateur, à cause de cet engagement spécial envers ce stakeholder, va négliger les autres groupes de stakeholders (Low et Srivatsan, 1995).

Troisièmement, le créateur peut relativiser les incertitudes par le choix du partenaire. Les incertitudes d'une relation d'échange sont aussi en corrélation avec la perception du stakeholder et avec ses capacités à réduire les incertitudes. Par conséquent, les créateurs doivent chercher des partenaires qui sont mieux en mesure d'absorber les conséquences d'une sousperformance, qui évaluent ces incertitudes comme étant plus faibles ou qui peuvent les réduire de manière relativement aisée. Par exemple, un investisseur qui possède le savoir-faire nécessaire pour vérifier les informations fournies par le créateur est confronté à des incertitudes plus faibles et a, par conséquent, des coûts de contrôle moins élevés. De tels partenaires accordent à ces incertitudes moins d'importance et seront plus disposés à entrer dans la relation d'échange offerte par le créateur.

Quatrièmement, le créateur peut aider le stakeholder à réduire les coûts qu'il devra assumer pour diminuer les incertitudes. Pour ce faire, le créateur fournira au stakeholder les informations nécessaires pour qu'il puisse vérifier leur exactitude et lui offrira les possibilités de contrôle correspondantes («entreprises transparentes»).

Cinquièmement, le créateur peut réduire pour le stakeholder les conséquences négatives des incertitudes. L'entrepreneur peut aussi réduire les incertitudes du stakeholder en assumant une partie du risque et en acceptant une rémunération au rendement (une contrepartie n'est versée qu'une fois que le créateur a démontré qu'il se comporte de manière convenue).

Sixièmement, le créateur peut essayer de transformer des «credence qualities» en «search qualities» ou en promesses crédibles (signaling; Kaas, 1992a; Kleinaltenkamp, 1992). Dans ce but, l'entrepreneur peut se référer aux rapports ou aux témoignages des premiers clients (Gruber, 2004), aux jugements de tiers (par exemple, expertises du plan d'affaires, alliances stratégiques), aux garanties de tiers (par exemple, une institution de subvention 
qui se porte garante pour un emprunt; Gruber, 2004) ou aux qualifications certifiées par des tiers (par exemple, certificats de formation ou prix; Gierl et Gehrke, 2004; Gruber, 2004).

Septièmement, le créateur peut faire des investissements élevés, qui peuvent être considérés comme des indicateurs de sa crédibilité. Les stakeholders sont conscients que dans des marchés transparents (!), seuls les entrepreneurs qui peuvent s'attendre à des rémunérations supérieures à la moyenne pour leurs performances supérieures à la moyenne peuvent prendre le risque d'investissements irrécupérables supérieurs à la moyenne (par exemple, des bureaux de standing élevé). Par conséquent, dans des marchés transparents, les investissements irrécupérables peuvent être vus comme un signe de qualité supérieure (Tolle, 1994).

Finalement, le créateur peut réduire sa propre marge de manœuvre. La spécificité des facteurs (d'input) qui doivent être mis en œuvre par l'entrepreneur (par exemple, la réalisation de certaines tâches par des employés particuliers ou l'utilisation de certains matériaux) limite la marge de manœuvre de l'entrepreneur en raison des procédures de surveillance (Kaas, 1992a). Avec la marge de manœuvre, les incertitudes du stakeholder diminuent.

\section{Les mesures créatrices de confiance dans la relation stakeholder-créateur}

Premièrement, le créateur peut implanter son entreprise dans un environnement qui lui est familier. Plus le créateur est connu, plus il sera facile pour le stakeholder de juger de sa fiabilité en prenant en compte son passé. Les créations d'entreprises qui s'appuient sur la notoriété du créateur dans son lieu d'origine se font plus facilement. Par conséquent, les réseaux personnels du créateur jouent un rôle important dans la phase de création de l'entreprise. Ils permettent à l'entrepreneur de surmonter le problème de la «liabilities of newness »- en particulier les déficits de crédibilité et de confiance (Gruber, 2004, en référence à Carson, 1985; Tyebjee, Bruno et McIntyre, 1983).

Deuxièmement, le créateur peut inspirer confiance. La confiance est liée au cycle de développement du comportement de l'entrepreneur perçu par les partenaires. Étant donné qu'il n'est possible de faire naître la confiance que par une application cohérente d'actions liées à des principes (maximes) stables dans la durée, l'entrepreneur est tenu de se comporter de manière constante. Compte tenu de la transparence des marchés, il est généralement impossible pour le créateur de présenter des images différentes de lui-même à différents stakeholders. La manière dont le créateur se présente devant un groupe de stakeholders atteste de la façon dont il est perçu par d'autres 
groupes de stakeholders (Jones, 1995). Ainsi, la gestion de l'information par le créateur a une grande importance autant pour la réduction des incertitudes que pour le développement de la confiance (Kollmann et Kuckertz, 2006b). «Open communication and dialogue are, in themselves, stakeholder benefits » (Preston, Donaldson et Brooks, 1999).

Finalement, le créateur peut se livrer volontairement aux stakeholders (= obligation envers lui-même) (Roessl, 1994, 1996, repris par Woratschek et Roth, 2003; Osterloh et Weibel, 2000; Sydow et Windeler, 2000; Gierl, 2000; Sydow, 1999; Wildemann, 1997). Par cet autoengagement volontaire, l'entrepreneur se rend dépendant du comportement du stakeholder et hausse ses propres coûts de défection. Par conséquent, le partenaire potentiel peut s'attendre à ce que le créateur réalise la performance convenue. De telles dépendances sont basées autant sur la réputation du créateur que sur les préperformances spécifiques qu'il apporte dans la relation. En ce qui concerne la réputation, le stakeholder peut nuire à celle-ci par diffamation (Kaas, 1992b; Spremann, 1988). En ce qui concerne les préperformances, il peut exploiter la problématique des coûts irrécupérables par le non-remboursement des préperformances déjà effectuées. Ces préperformances volontaires rendent l'offre plus intéressante pour le stakeholder potentiel. De plus, ces autoengagements démontrent de manière convaincante l'aptitude du créateur à effectuer la performance convenue. Finalement, la vulnérabilité de l'entrepreneur, provoquée délibérément, est le point de départ de l'évolution d'une relation de confiance. Le créateur a confiance que le stakeholder n'exploitera pas les possibilités d'opportunisme créées par le créateur lui-même par son autoengagement, et ce n'est que grâce à cette confiance - au début unilatérale qu'une relation basée sur la confiance peut s'installer (Luhmann, 1989). Ainsi se manifeste le pouvoir coordinateur des préperformances risquées, même si ce pouvoir n'est pas strictement coordinateur: si une action d'un acteur est considérée comme amicale, on se sent obligé de réagir de la même manière (Irlenbusch et Schade, 1999).

Sous l'angle de l'approche principal-agent, nous avons analysé la création d'entreprise et la relation stakeholder-créateur. La présente contribution a accentué le rôle crucial des incertitudes en phase de start-up. Nous avons ensuite examiné, sur la base de la théorie des coûts de transaction, les principaux moyens pouvant réduire les incertitudes dans le contexte de la création d'entreprise. Ces considérations ont été associées à un outil managérial dans l'approche MUT. Malgré des propositions de mesures très concrètes, nous devons souligner le fait que des relations avec les stakeholders basées sur la confiance ne peuvent être développées que progressivement à travers un lent processus de réalisation des facteurs qui les rassureront. 


\section{Bibliographie}

AdLer, P.S. (2001), «Market, hierarchy, and trust: the knowledge economy and the future of capitalism», Organization Science, vol. 12, n 2, p. 215-234.

Barnard, C.I. (1938), The Functions of the Executive, Cambridge, Mass., Harvard University Press.

BECKER, C. (2001), «Oekonomische Analyse neoklassischer vertraege: funktion und wirkweise von schiedsrichtern und schlichtern in komplexen transaktionsbeziehungen », Zeitschrift fuer betriebswirtschaftliche Forschung, vol. 53, p. 39-54.

Berman, S.L., A.C. Wicks, S. Kotha et T.M. Jones (1999), «Does stakeholder orientation matter? The relationship between stakeholder management models and firm financial performance», Academy of Management Journal, vol. 42, $\mathrm{n}^{\circ}$ 5, p. 488-506.

BreID, V. (1995), «Aussagefaehigkeit agencytheoretischer Ansaetze im Hinblick auf die Verhaltenssteuerung von Entscheidungstraegern », Zeitschrift fuer betriebswirtschaftliche Forschung, vol. 47, p. 821-854.

Brouthers, K.D. et G. NAKos (2004), «SME entry mode choice and performance: a transaction cost perspective», Entrepreneurship, Theory \& Practice, vol. 28, n 3 , p. 229-247.

CARson, D.J. (1985), «The evolution of marketing in small firms », European Journal of Marketing, vol. 19, p. 7-16.

CARSON, S.J.,A. MAdHOK et T.Wu (2006), «Uncertainty, opportunism and governance: the effects of volatility and ambiguity on formal and relational contracting», Academy of Management Journal, vol. 49, n 5, p. 1058-1077.

CHor, Y.R. (2001), The Early Life of a New Venture: An Analysis of Entrepreneurs' Strategic Decisions and Stakeholders'Assessment, New York, Dissertation at the Rensselaer Polytechnic Institute Troy.

Dwyer, F.R., P.H. SchurR et S. OH (1987), «Developing buyer-seller relationships », Journal of Marketing, vol. 51, n 2, p. 11-27.

EBerl, P. (2004), «The development of trust and implications for organizational design: a game- and attribution-theoretical framework », Schmalenbach Business Review, vol. 56, n 3 , p. 258-273.

Fallgatter, M.J. (2004), «Entrepreneurship: Konturen einer jungen Disziplin», Zeitschrift fuer betriebswirtschaftliche Forschung, vol. 56, p. 23-44.

FINK, M. (2005), Selbstverpflichtung als Erfolgsfaktor bei langfristigen, komplexen Transaktionsbeziehungen, Frankfurt, Wien, Peter Lang.

Frederick, W.C., J.E. Post et K. Davis (1992), Business and Society: Corporate Strategy, Public Policy, Ethics, $7^{\mathrm{e}}$ éd., New York, McGraw-Hill.

Freeman, R.E. (1984), Strategic Management: A Stakeholder Approach, Boston, Mass., Pitman. 
Freeman, R.E. et J. McVea (2005), «A stakeholder approach to strategic management», dans M.A. Hitt, R.E. Freeman et J.S. Harrison (dir.), The Blackwell Handbook of Strategic Management, Malden, Mass., Blackwell, p. 189-207.

Freeman, R.E. et R.A. Phillips (2002), «Stakeholder theory: a libertarian defense», Business Ethics Quarterly, vol. 12, n 3, p. 331-349.

Frey, B.S. et M. Osterloh (2002), Successful Management by Motivation: Balancing Intrinsic and Extrinsic Incentives, Berlin, Heidelberg.

Gaechter, S., B. Herrmann et C. Thoeni (2004), «Trust, voluntary cooperation, and socio-economic background: survey and experimental evidence », Journal of Economic Behaviour \& Organization, vol. 55, p. 505-531.

Gierl, H. (2000), «Opportunismus in langfristigen Geschaeftsbeziehungen», Zeitschrift fuer betriebswirtschaftliche Forschung, vol. 52, p. 107-140.

Gierl, H. (2001), «Opportunismus in Geschaeftsbeziehungen - Ursachen und Gegenmassnahmen », Der Markt, vol. 40/157-158/55-65.

Gierl, H. et S. BAmbauer (2002), «Information networks as a safeguard from opportunism in industrial supplier-buyer relationships », Schmalenbach Business Review, vol. 54, n 4, p. 335-350.

Gierl, H. et G. GeHrKe (2004), «Kundenbindung in industriellen Zuliefer-AbnehmerBeziehungen», Zeitschrift fuer betriebswirtschaftliche Forschung, vol. 56, p. 203-236.

GrichNiK, D. et F. SCHWAERZEL (2005), «Gruendungsfinanzierung und Transaktionskostentheorie», WiSt, vol. 11, p. 613-618.

Griesinger, D.W. (1990), «The human side of economic organization », Academy of Management Review, vol. 15, p. 478-499.

Gruber, M. (2004), «Marketing in new ventures: theory and empirical evidence», Schmalenbach Business Review, vol. 56, n 2, p. 164-199.

Harvey, S.J. (2002), «The trust paradox: a survey of economic inquiries into the nature of trust and trustworthiness », Journal of Economic Behavior \& Organization, vol. 47, p. 291-307.

Hauschildt, J. et J. Leker (1990), «Flexibilisierung als Strategie von Anbietern und Nachfragern innovativer Gueter», Zeitschrift fuer betriebswirtschaftliche Forschung, vol. 42, p. 963-975.

Irlenbusch, B. et L. Schade (1999), «Zur Wirksamkeit nicht bindender Vertraege - eine experimentelle Untersuchung», Zeitschrift fuer betriebswirtschaftliche Forschung, vol. 51, p. 730-752.

JONES, G.R. (2005), «Towards a positive interpretation of transaction cost theory: the central roles of entrepreneurship and trust », dans M.A. Hitt, R.E. Freeman et J.S. Harrison (dir.), The Blackwell Handbook of Strategic Management, Malden, Mass., Blackwell, p. 208-228.

JoNES, T.M. (1995), «Instrumental stakeholder theory: a synthesis of ethics and economics », Academy of Management Review, vol. 20, n 2, p. 404-437. 
KAAs, K.P. (1992a), «Kontraktguetermarketing als Kooperation zwischen Prinzipalen und Agenten », Zeitschrift fuer betriebswirtschaftliche Forschung, vol. 44, p. 884-901.

KaAs, K.P. (1992b), Marketing und Neue Institutionenlehre, Document de travail, Frankfurt, University of Frankfurt.

KleinaltenKAmP, M. et F. JАCOB (2002), «German approaches to business-to-business marketing theory - Origins and structure», Journal of Business Research, vol. 55, p. 149-155.

Kleinaltenkamp, M. (1992), «Investitionsgueter-Marketing aus informationsoekonomischer Sicht», Zeitschrift fuer betriebswirtschaftliche Forschung, vol. 44, p. 809-829.

Kollmann, T. et A. KuCKertz (2005), «Rahmenbedingungen der Unternehmensgruendung - eine Analyse des „Entrepreneurial Events“ im internationalen Vergleich », Betriebswirtschaftliche Forschung und Praxis, vol. 57, $\mathrm{n}^{\circ}$ 4, p. 307-318.

Kollmann, T. et A. KucKertz (2006a), «Venture archetypes and the entrepreneurial event: cross-cultural empirical evidence», Journal of Enterprising Culture, vol. 14, n ${ }^{\circ}$, p. 27-48.

Kollmann, T. et A. KuCKertz (2006b), «Investor relations for start-ups : an analysis of venture capital investor's communicative needs », International Journal of Technology Management, vol. 34, $\mathrm{n}^{\text {os }} 1-2, \mathrm{p} .47-62$.

LEVITT, Th. (1981), «Marketing intangible products and product intangibles », Harvard Business Review, vol. 59, n 4, p. 94-102.

LindsKold, S. (1981), «Die Entwicklung von Vertrauen, der GRIT-Ansatz und die Wirkung von konziliantem Handeln auf Konflikt und Kooperation », dans W. Grunwald et H.-G. Lilge (dir.), Kooperation und Konkurrenz in Organisationen, Bern, Stuttgart, Paul Haupt, p. 241-273.

Low, M.B. et V. SRIVATSAN (1995), «What does it mean to trust an entrepreneur?», dans S. Birley et I.C. MacMillan (dir.), International Entrepreneurship, Londres, Routledge, p. 59-78.

Luhmann, N. (1989), Vertrauen, Ein Mechanismus der Reduktion sozialer Komplexitaet, $3^{\mathrm{e}}$ éd., Stuttgart, Enke.

March, J.G. et H.A. Simon (1958), Organizations, New York, John Wiley \& Sons.

Osterloh, M. et A. Weibel (2000), «Ressourcensteuerung in Netzwerken: Eine Tragoedie der Allmende? », dans J. Sydow et A. Windeler (dir.), Steuerung von Netzwerken, Opladen, Westdeutscher Verlag, p. 88-106.

Phillips, R., R.E. Freeman et A.C. Wicks (2003), «What stakeholder theory is not», Business Ethics Quarterly, vol. 13, n 4, p. 479-502.

Picot, A. (1991), «Ein neuer Ansatz zur Gestaltung der Leistungstiefe», Zeitschrift fuer betriebswirtschaftliche Forschung, vol. 43, p. 336-357. 
Powell, J.D. et C.F. Bimmerle (1980), «A model of entrepreneurship: moving toward precision and complexity», Journal of Small Business Management, vol. 18, $\mathrm{n}^{\mathrm{o}} 1, \mathrm{p} .33-36$.

Preston, L.E., T. Donaldson et L.J. Brooks (1999), «Principles of stakeholder management», dans L.E. Preston, T. Donaldson et L.J. Brooks (dir.), Principles of Stakeholder Management, Toronto, Clarkson Centre of Business Ethics, Joseph L. Rotman School of Management, University of Toronto, p. 1-8.

Provan, K.G. et St.J. Skinner (1989), «Interorganizational dependence and control as predictors of opportunism in dealer-supplier relations », Academy of Management Review, vol. 32, p. 202-212.

RAPPAPORT, A. (1995), Shareholder Value:Wertsteigerung als Massstab fuer die Unternehmensfuehrung, Stuttgart, Schaeffer-Poeschel.

RoessL, D. (1994), Gestaltung komplexer Austauschbeziehungen - Analyse zwischenbetrieblicher Kooperation, Wiesbaden, Gabler.

Roessl, D. (1996), «Selbstverpflichtung als alternative Koordinationsform von komplexen Austauschbeziehungen », Zeitschrift fuer betriebswirtschaftliche Forschung, vol. 48, p. 311-334.

Roessl, D. (2006), Relationship Management - mit Beitraegen aus Wissenschaft und Praxis, Wien, facultas.

Schrader, St. (1993), «Kooperation», dans J. Hauschildt et O. Gruen (dir.), Ergebnisse empirischer betriebswirtschaftlicher Forschung - Zu einer Realtheorie der Unternehmung, Festschrift fuer Eberhard Witte, Stuttgart, Schaeffer Poeschel, p. 221-254.

Semlinger, K. (1993), «Effizienz und Autonomie in Zuliefernetzwerken: zum strategischen Gehalt von Kooperation», dans W.H. Staehle et J. Sydow (dir.), Managementforschung 3, Berlin, New York, Walter de Gruyter, p. 309-354.

SIEgrist, M. (2001), Die Bedeutung von Vertrauen bei der Wahrnehmung und Bewertung von Risiken, Zuerich, Universitaet Zuerich.

Spremann, K. (1990), «Asymmetrische Information», Zeitschrift fuer Betriebswirtschaft, vol. 60, p. 561-586.

Spremann, K. (1988), «Reputation, garantie, information», Zeitschrift fuer Betriebswirtschaft, vol. 58, p. 613-629.

Sydow, J. (1999), «Quo Vadis Transaktionskostentheorie? Wege, Irrwege, Auswege», dans Th. Edeling, W. Jann et D. Wagner (dir.), Institutionenoekonomie und Neuer Institutionalismus, Schriftenreihe Interdisziplinaere Organisations- und Verwaltungsforschung 2, Opladen, Leske + Buderich, p. 165-176.

Sydow, J. et A. Windeler (2000), «Steuerung von und in Netzwerken: Perspektiven, Konzepte, vor allem aber offene Fragen », dans J. Sydow et A. Windeler (dir.), Steuerung von Netzwerken: Konzepte und Praktiken, Wiesbaden, Opladen, p. 1-24. 
Tolle, E. (1994), «Informationsoekonomische Erkenntnisse fuer das Marketing bei Qualitaetsunsicherheit der Konsumenten», Zeitschrift fuer betriebswirtschaftliche Forschung, vol. 46, p. 926-938.

Tyebjee, T.T., A.V. Bruno et S.H. McIntyre (1983), «Growing ventures can anticipate marketing stages », Harvard Business Review, vol. 61, nº 1, p. 62-66.

Weiber, R. et J. Adler (1995), «Informationsoekonomisch begruendete Typologisierung von Kaufprozessen », Zeitschrift fuer betriebswirtschaftliche Forschung, vol. 47, p. 43-65.

Wheeler, D., B. Colbert, B. et R.E. Freeman (2003), «Focusing on value: reconciling corporate social responsibility, sustainability and a stakeholder approach in a network world », Journal of General Management, vol. 28, n 3, p. 1-28.

WiLdEMANN, H. (1997), «Koordination von Unternehmensnetzwerken», Zeitschrift fuer Betriebswirtschaft, vol. 67, n 4, p. 417-439.

Williamson, O.E. (1981), «The economics of organization: the transaction cost approach », American Journal of Sociology, vol. 87, p. 548-577.

Williamson, O.E. (1991), «Comparative economic organization. Vergleichende oekonomische Organisationstheorie: Die Analyse diskreter Strukturalternativen », dans D. Ordelheide, B. Rudolph et E. Buesselmann (dir.), Betriebswirtschaftslehre und Oekonomische Theorie, Stuttgart, Poeschel, p. 13-49.

Williamson, O.E. (2000), «The new institutional economics: taking stock, looking ahead», Journal of Economic Literature, vol. 38, n 3, p. 595-613.

Williamson, O.E. (2002), «The lens of contract: private ordering», The American Economic Review, vol. 92, n 2, p. 438-443.

Williamson, O.E. (2003), «Examining economic organization through the lens of contract», Industrial and Corporate Change, vol. 12, n 4, p. 917-942.

Williamson, O.E. (2005), «The economics of governance», AEA Papers and Proceedings, vol. $95, \mathrm{n}^{\circ} 2$, p. 1-18.

WoratscheK, H. et St. Roth (2003), «Kooperation: Erklaerungsperspektive der Neuen Institutionenoekonomik», dans J. Zentes, B. Swoboda et D. Morschett (dir.), Kooperationen, Allianzen und Netzwerke, Wiesbaden, Gabler, p. 141-166. 